\title{
Light Echoes of Historic Transients
}

\author{
Armin Rest ${ }^{1}$, B. Sinnott ${ }^{2}$, D. L. Welch ${ }^{2}$, J. L. Prieto ${ }^{3}$ and F. Bianco ${ }^{4}$ \\ ${ }^{1}$ STScI, 3700 San Martin Dr., Baltimore, MD 21218, USA \\ email: arest@stsci.edu \\ ${ }^{2}$ Department of Physics and Astronomy, McMaster University, \\ Hamilton, Ontario L8S 4M1, Canada \\ ${ }^{3}$ Department of Astrophysical Sciences, Princeton University, \\ 4 Ivy Lane, Princeton, NJ 08544, USA \\ ${ }^{4}$ Center for Cosmology and Particle Physics, New York University, \\ 4 Washington Place, New York, NY 10003, USA
}

\begin{abstract}
Light echoes, light from a variable source scattered off dust, have been observed for over a century. The recent discovery of light echoes around centuries-old supernovae in the Milky Way and the Large Magellanic Cloud have allowed the spectroscopic characterization of these events, even without contemporaneous photometry and spectroscopy using modern instrumentation. Here we review the recent scientific advances using light echoes of ancient and historic transients, and focus on our latest work on SN 1987A's and Eta Carinae's light echoes.
\end{abstract}

Keywords. reflection nebulae, supernovae: general, supernovae: individual (SN 1987A), stars: individual ( $\eta$ Car), stars: variables: other

\section{Introduction}

Light echoes (LEs) arise when light from a transient or variable source is scattered off circumstellar or interstellar dust, reaching the observer after a time delay resulting from the longer path length (e.g., Couderc 1939; Chevalier 1986; Schaefer 1987; Xu et al. 1994; Sugerman 2003; Patat 2005). Over a century ago, in 1901, the first scattered LEs were discovered around Nova Persei (Ritchey 1901a,b, 1902). They were recognized as such shortly thereafter by Kapteyn (1902) and Perrine (1903). Since then, LEs have been observed around a wide variety of objects: the Galactic Nova Sagittarii 1936 (Swope 1940), the eruptive variable V838 Monocerotis (Bond et al. 2003), the Cepheid RS Puppis (Westerlund 1961; Havlen 1972), the T Tauri star S CrA (Ortiz et al. 2010), and the Herbig Ae/Be star R CrA (Ortiz et al. 2010). Echoes have also been observed from extragalactic SNe, with SN 1987A being the most famous case (Crotts 1988; Suntzeff et al. 1988b), but also including SNe 1980K (Sugerman et al. 2012), 1991T (Schmidt et al. 1994; Sparks et al. 1999), 1993J (Sugerman \& Crotts 2002; Liu et al. 2003), 1995E (Quinn et al. 2006), 1998bu (Garnavich et al. 2001; Cappellaro et al. 2001), 2002hh (Welch et al. 2007; Otsuka et al. 2012), 2003gd (Sugerman 2005; Van Dyk et al. 2006; Otsuka et al. 2012), 2004et (Otsuka et al. 2012), 2006X (Wang et al. 2008; Crotts \& Yourdon 2008), 2006bc (Gallagher et al. 2011; Otsuka et al. 2012), 2006gy (Miller et al. 2010), 2007it (Andrews et al. 2011), and 2008bk (Van Dyk 2013). All of the aforementioned LEs had the common selection criterion that they were found serendipitously while the transient source was still bright.

Early on in the last century, Zwicky (1940) had the idea that it might be possible to learn more about historical SNe by studying their scattered LEs. However, the few dedicated surveys trying to implement this idea for historic SNe (van den Bergh 1965a,b, 1966; Boffi et al. 1999) and novae (van den Bergh 1977; Schaefer 1988) were not suc- 
cessful. With the emergence of CCDs as astronomical detectors in combination with the advancement in telescope technology that allowed to image larger field-of-views, the wide-field time-domain surveys at the beginning of this century significantly improved in depth and area. These improvements led to the first discoveries of LEs at angular distances from the ancient transients too large to suggest an immediate association. The i400-900 year-old LEs from three LMC supernovae were found by Rest et al. (2005b) as part of the SuperMACHO survey (Rest et al. 2005a). Subsequent targeted searches in our Galaxy found LEs of Tycho's SN (Rest et al. 2007, 2008b), Cas A (Rest et al. 2007, 2008b; Krause et al. 2008a), and $\eta$ Carinae (Rest et al. 2012a).

\section{Light Echo Spectroscopy}

Spectroscopy of LEs allows the transient to be studied long after it has already faded. The first LE spectrum was a 35 hour exposure of one of Nova Persei 1901 LEs (Perrine 1903), confirming that the nebulous moving features seen around Nova Persei were indeed its echoes. LE spectra of SN 1987A were most similar to those of the SN near maximum light (Gouiffes et al. 1988; Suntzeff et al. 1988b). Serendipitously, Schmidt et al. (1994) found the spectrum of SN $1991 \mathrm{~T}$ taken 750 days after maximum again similar to the one at peak, indicating that at that time echoes from the SN at peak dominated the spectrum.

Spectroscopy of ancient SN LEs discovered in the LMC (Rest et al. 2005b) resulted in the first opportunity to classify ancient transients long after their direct light had encountered Earth (Rest et al. 2008a). The subsequent discovery and spectroscopy of LEs of Cas A and Tycho (Rest et al. 2007, 2008b; Krause et al. 2008a,b) allowed their spectroscopic classification as a SN IIb and normal SN Ia, respectively.

At first, it was widely believed that an observed LE spectrum would be the lightcurveweighted integration of the transients' individual epochs (e.g., Patat et al. 2006; Rest et al. 2008a; Krause et al. 2008a). Such an integration is equivalent to assuming that the dust filament is thick, which is not always the case(Rest et al. 2011b, 2012b). Spectral features which persist for long periods of time during the evolution of an outburst will be weighted much more strongly when scattered by a thick filament than a thin filament.

In addition to spectroscopic classification, LEs also offer two more exciting scientific opportunities: "3D spectroscopy and "spectroscopic time series" of transients. Examples of realization of these techniques are provide in the following two sections using SN 1987A and $\eta$ Carinae.

\subsection{D Spectroscopy}

LEs scattered by different dust structures offer an opportunity that is unique in astronomy: probe the same object directly from different directions. Fig. 1 shows the light paths of seven SN 1987A LEs. By observing these LEs, SN 1987A can be analyzed as if the observers are at different line-of-sights, allowing to directly compare different hemispheres of one and the same object. This technique was first applied to observe $\eta$ Carinae central star from different directions using spectra of the reflection nebula (Boumis et al. 1998; Smith et al. 2003).

Rest et al. (2011a) obtained spectra of three different Cas A LEs, viewing the Cas A SN from very different lines-of-sight. After accounting for the effects of the scattering dust, they found that the He I $\lambda 5876$ and $\mathrm{H} \alpha$ features of one LE are blue-shifted by an additional $\sim 4000 \mathrm{~km} \mathrm{~s}^{-1}$ relative to the other two LE spectra. X-ray and optical data of the Cas A remnant also show a Fe-rich outflow in the same direction (Burrows et al. 2005; Wheeler et al. 2008; DeLaney et al. 2010). This indicates that Cas A was an intrinsically 


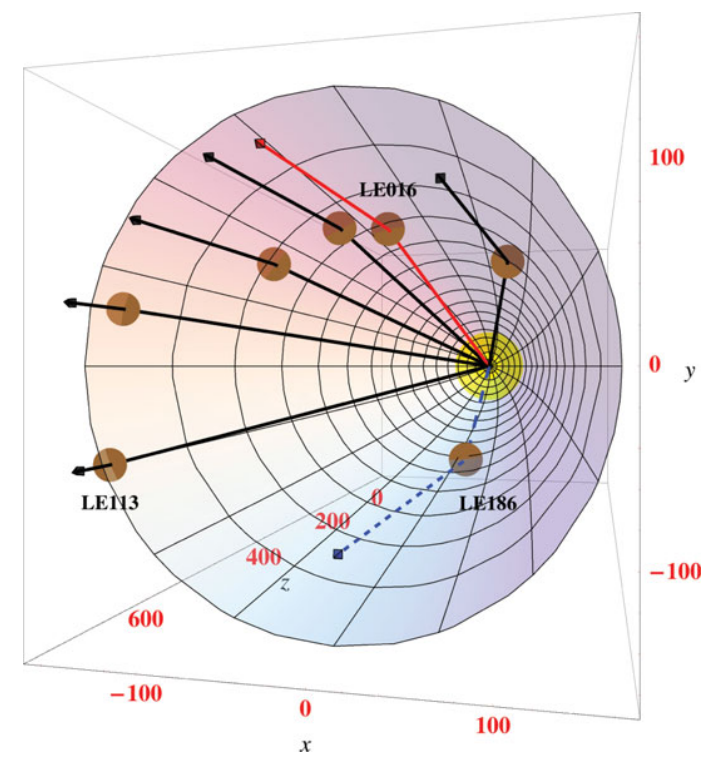

Figure 1. Light paths and 3D scattering dust locations for seven LEs of SN 1987A spectroscopically observed by Sinnott et al. (2013). Solid red and dashed blue lines highlight the extreme north and south viewing angles corresponding to LE016 and LE186 (see Figure 2). North is towards the positive $\mathrm{y}$ axis, east is towards the negative $\mathrm{x}$ axis, and $\mathrm{z}$ is the distance in front of the SN. All units are in light years. This figure is courtesy of Sinnott et al. (2013).

asymmetric SN. The blue-shifted SN ejecta is in the direction approximately opposite the motion of the resulting neutron star, suggesting that the explosion mechanism that gave the neutron star its kick affected the outer layers of the SN. This appears to be the first instance where the structure of the SN remnant can be directly associated with asymmetry observed in the explosion itself.

SN 1987A has a very rich set of LEs scattering off circumstellar (Crotts \& Kunkel 1991; Crotts et al. 1995; Sugerman et al. 2005a,b) and interstellar dust (Crotts 1988; Suntzeff et al. 1988b; Gouiffes et al. 1988; Couch et al. 1990; Xu et al. 1994, 1995). The LEs shown in Figure 1 were spectroscopically observed by Sinnott et al. (2013). After correcting for the effects of the scattering dust, these spectra can be directly compared to a LE spectrum constructed from the spectro-photometric library of SN 1987A (Menzies et al. 1987; Catchpole et al. 1987, 1988; Hamuy et al. 1988; Suntzeff et al. 1988a; Whitelock et al. 1988; Catchpole et al. 1989; Phillips et al. 1988, 1990). Sinnott et al. (2013) find an excess in redshifted $\mathrm{H} \alpha$ emission and a blueshifted knee for the LE LE016 at position angle $\mathrm{PA}=16^{\circ}$ (see red line in Figure 2). Both asymmetry signatures disappear as the $\mathrm{PA}$ increases, and then reappear in the form of an excess in blueshifted $\mathrm{H} \alpha$ emission and a redshifted knee at the opposite $\mathrm{PA}=186^{\circ} \mathrm{LE}, \mathrm{LE} 186$ (see blue line in Figure 2).

In Figure 3, the light paths of the LE186 and LE016 echoes are illustrated and compared to the structure of the SN 1987A ejecta (Kjær et al. 2010). Even though the opening angle of the two LEs is only $\sim 40^{\circ}$, the differences in the $\mathrm{H} \alpha$ lines are quite striking. Sinnott et al. (2013) argues that these differences are caused by a two-sided and asymmetric ${ }^{56} \mathrm{Ni}$ distribution in the outer $\mathrm{H}$ envelope. The symmetry axis defined by the $16^{\circ} / 186^{\circ}$ viewing angles is in excellent agreement with the PA of the symmetry axis of the elongated ejecta that was measured to be $\sim 15^{\circ}$ (Wang et al. 2002; Kjær et al. 2010). Kjær et al. (2010) also found the present-day ejecta to be blueshifted in the north and redshifted in the south, inclined out of the plane of the sky by $\sim 25^{\circ}$. The two-sided ${ }^{56} \mathrm{Ni}$ 


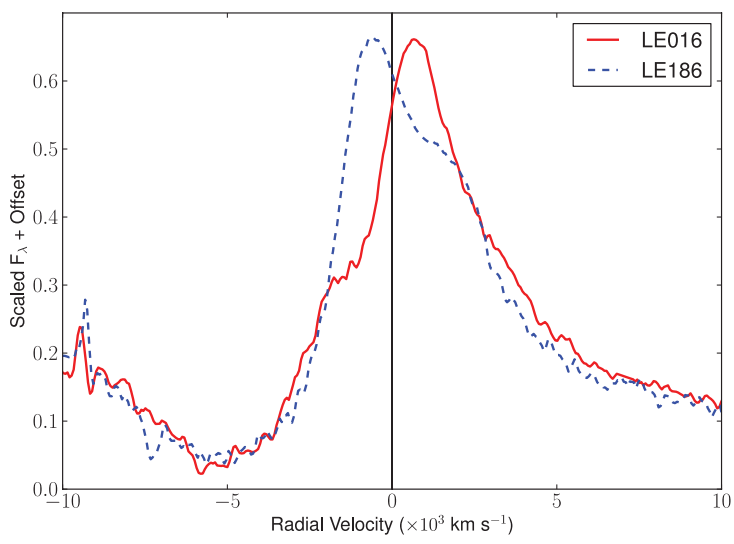

Figure 2. Observed $\mathrm{H} \alpha$ lines from LE016 and LE186. Emission peaks have been interpolated with high-order polynomials. Spectra are scaled and offset for comparison purposes, as well as smoothed with a boxcar of 3 pixels. Although this plot does not take into account the important differences in LE time-integrations between the spectra, it highlights the overall difference in fine-structure in the two LE spectra from opposite PAs. Observing $\mathrm{H} \alpha$ profiles with opposite asymmetry structure at opposite PAs is surprising considering the opening angle between the two LEs is $<40^{\circ}$. This figure and caption is courtesy of Sinnott et al. (2013).
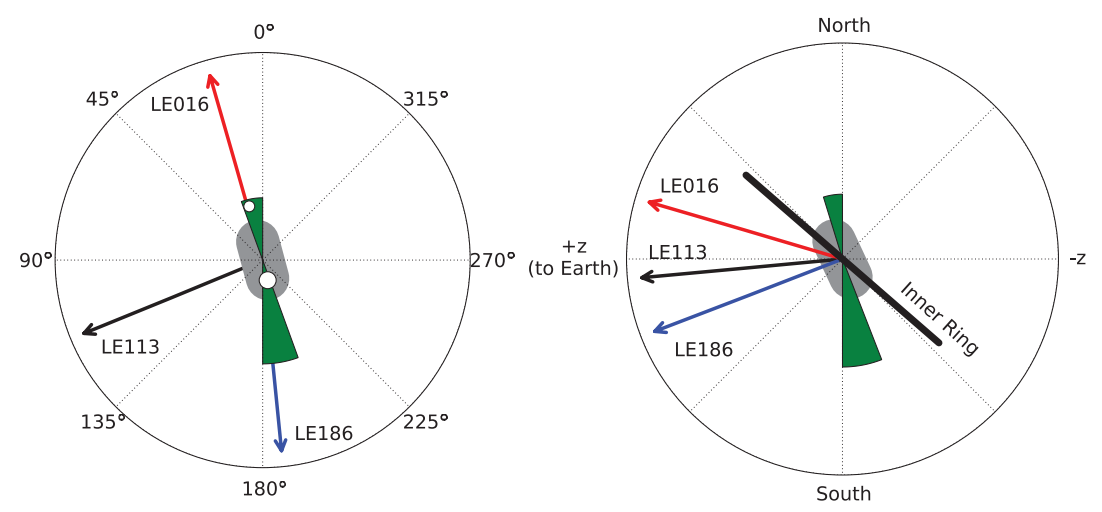

Figure 3. LEFT: PAs on the sky of the three dominant LE viewing angles LE016, LE113 and LE186. The central grey region denotes the orientation on the sky of the elongated remnant ejecta $\left(\mathrm{PA}=16^{\circ}\right)$ from Kjær et al. $(2010)$. The green wedges illustrate the proposed two-sided distribution of ${ }^{56} \mathrm{Ni}$, most dominant in the southern hemisphere. White circles illustrate the locations of the two mystery spots as identified by Nisenson \& Papaliolios (1999), with radius proportional to relative brightness in magnitudes of the two sources. Only the relative distance from the center of the SNR to the mystery spots is to scale in the image. RIGHT: Schematic with viewing angle perpendicular to Earths line of sight. The inclination of the inner circumstellar ring is shown along with the proposed two-sided distribution of ${ }^{56} \mathrm{Ni}$ in green. Note that the green wedges are to highlight the proposed geometry (not absolute velocity) of the ${ }^{56} \mathrm{Ni}$ asymmetry probed by the LE spectra, illustrating that the southern overabundance is most dominant. This figure and caption is courtesy of Sinnott et al. (2013).

distribution proposed by Sinnott et al. (2013) as an explanation for the asymmetry seen in the LE observations is therefore roughly aligned with the $\sim 25$-year-old emerging SN remnant both in $\mathrm{PA}$ and inclination out of the sky.

\subsection{Spectroscopic Time Series}

If the scattering dust filament is infinitely thin, then the LE is just the projected light curve of the transient. Therefore it is, in theory, possible to obtain spectra from different 

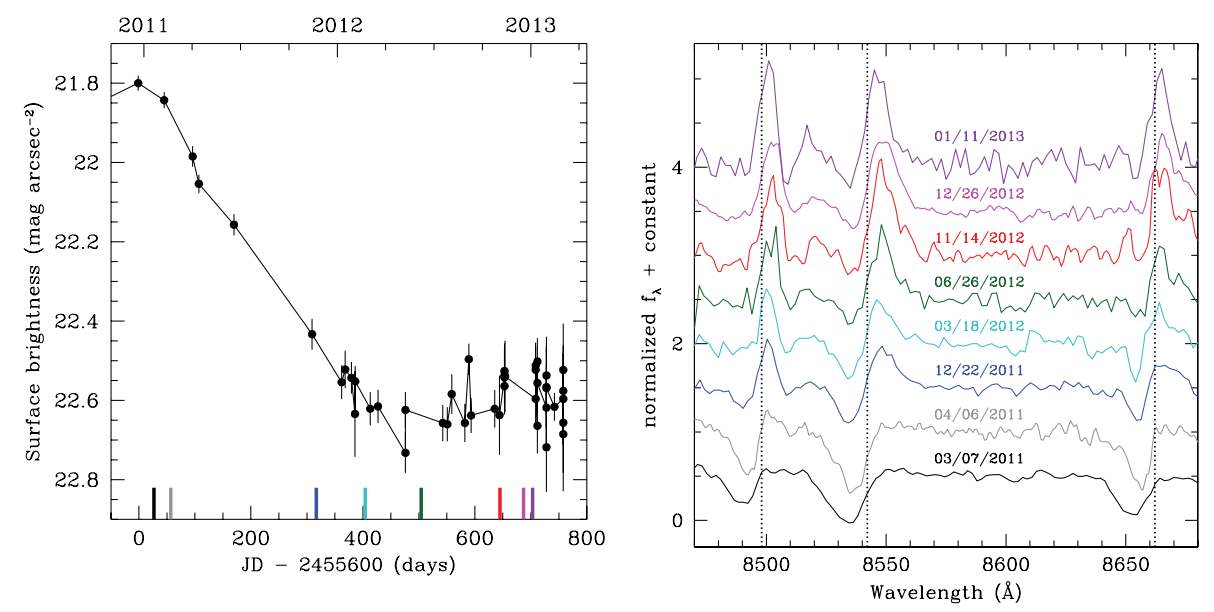

Figure 4. LEFT: Light curve of part of $\eta$ Car's Great Eruption, derived from its LEs. Shown is the surface brightness in $i$ band from the same sky position at different epochs (Blanco $4 \mathrm{~m}$ (MOSAIC II, DECam), Swope (Direct CCD), FTS (Spectral), SOAR (SOI)). The LE is the projected light curve of the source transient. Since the LE has an apparent motion, the light curve of the source event "moves" through a given position on the sky. The epochs spectra were taken are indicated with the colored lines. RIGHT: LE spectra of $\eta$ Car's Great Eruption showing the Ca II IR triplet from different epochs at the same sky location (Magellan Baade (IMACS), Gemini-S (GMOS)).

epochs of an event. In practice, there are complications: The LE profile gets convolved by the finite thickness of the scattering dust filament and the finite size of their slit and PSF at the time of observation (Rest et al. 2011b, 2012b). For typical Galactic LEs of ancient SNe and typical dust structures, the spatial extent is on the order of arcseconds and the temporal resolution ranges from time scales of a week under the most favorable circumstances, to months if the scattering dust filament is thick and/or unfavorably inclined. This means that SNe LEs, which have time-scales of a couple of months, can only be temporally resolved for very thin dust filaments and under excellent seeing conditions from the ground or space. However, transients with much longer time scales can be resolved much easier. One example is the Great Eruption of $\eta$ Car, which lasted two decades and showed temporal variability on time-scales of months. In this case, LE spectra from different epochs are only marginally affected by dust width, slit and PSF size.

The left panel of Figure 4 shows the LE flux of $\eta$ Car's Great Eruption at a given RA and Dec for various epochs. The light curve "moves" through a given position, and the shown flux is the light curve of some part of the Great Eruption convolved with the scattering dust thickness. The colored vertical lines indicate when we took a spectrum of the LE. The right panel of Figure 4 shows the corresponding spectrum in the same colors for the wavelength range covering the Ca II IR triplet. The spectrum taken close to the peak in the light curve correlates best with spectra of G2-to-G5 supergiants, a later range of types than predicted by standard opaque wind models (Rest et al. 2012a). The Ca II IR triplet is in absorption with an average blueshift of $200 \mathrm{~km} \mathrm{~s}^{-1}$, and the lines are asymmetric extending up to blueshifts of $800 \mathrm{~km} \mathrm{~s}^{-1}$ (Rest et al. 2012a). Spectra taken at later epochs during the declining part of the light curve show a transition of the Ca II IR triplet from pure absorption through a P-Cygni profile to a nearly pure emission line spectrum. In addition, strong CN molecular bands develop (see Figure 5). This is unlike other LBV outbursts which move back to the earlier stellar types toward 


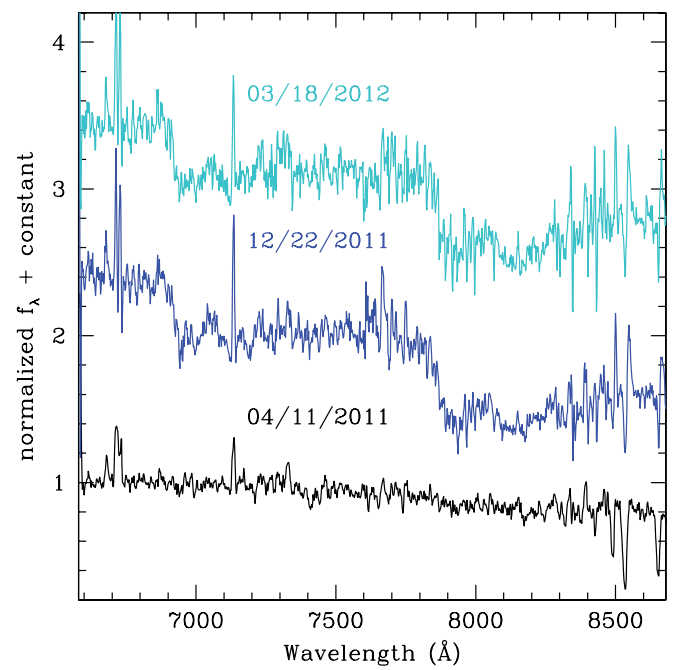

Figure 5. LE spectra of $\eta$ Car's Great Eruption showing how the CN molecular bands develop for later epochs.

the end of the eruptions. The LEs of $\eta$ Car indicate that the Great Eruption was not a typical LBV giant eruption. A paper detailing these observations is in preparation.

\section{Summary}

In the last decade, LE spectroscopy has emerged as powerful tool to spectroscopically classify ancient and historic SNe, for which no contemporary observations with modern instrumentation were possible. More recently, the technique of LE spectroscopy has been refined and improved, and it is now possible to utilize it to directly probe the asymmetries of the transient sources of the LEs. Furthermore, a spectroscopic time series can be obtained for favorable scattering dust filament structure or if the source transient is an event with a long time-scale.

\section{Acknowledgments}

We thank all the observers that have contributed to the monitoring of $\eta$ Car's LEs, especially E. Hsiao (and the Carnegie Supernova Project II) and T. Matheson. Based on observations of program GS-2012B-Q-57 obtained at the Gemini Observatory, which is operated by the Association of Universities for Research in Astronomy, Inc., under a cooperative agreement with the NSF on behalf of the Gemini partnership: the National Science Foundation (United States), the National Research Council (Canada), CONICYT (Chile), the Australian Research Council (Australia), Ministério da Ciência, Tecnologia e Inovação (Brazil) and Ministerio de Ciencia, Tecnología e Innovación Productiva (Argentina). This paper includes data gathered with the 6.5 meter Magellan Telescopes located at Las Campanas Observatory, Chile. Based on observations at the Cerro Tololo Inter-American Observatory, National Optical Astronomy Observatory, which are operated by the Association of Universities for Research in Astronomy, under contract with the National Science Foundation. The SOAR Telescope is a joint project of: Conselho Nacional de Pesquisas Cientificas e Tecnologicas CNPq-Brazil, The University of North Carolina at Chapel Hill, Michigan State University, and the National Optical Astronomy Observatory. 


\section{References}

Andrews, J. E., et al. 2011, ApJ, 731, 47

Boffi, F. R., Sparks, W. B., \& Macchetto, F. D. 1999, A\&AS, 138, 253

Bond, H. E., et al. 2003, Nature, 422, 405

Boumis, P., Meaburn, J., Bryce, M., \& Lopez, J. A. 1998, MNRAS, 294, 61

Burrows, A., Walder, R., Ott, C. D., \& Livne, E. 2005, in Astronomical Society of the Pacific Conference Series, Vol. 332, The Fate of the Most Massive Stars, ed. R. Humphreys \& K. Stanek, 350-+

Cappellaro, E., et al. 2001, ApJ, 549, L215

Catchpole, R. M., et al. 1987, MNRAS, 229, 15P

- . 1988, MNRAS, 231, 75P 1989, MNRAS, 237, 55P

Chevalier, R. A. 1986, ApJ, 308, 225

Couch, W. J., Allen, D. A., \& Malin, D. F. 1990, MNRAS, 242, 555

Couderc, P. 1939, Annales d'Astrophysique, 2, 271

Crotts, A. 1988, IAU Circ., 4561, 4

Crotts, A. P. S. \& Kunkel, W. E. 1991, ApJ, 366, L73

Crotts, A. P. S., Kunkel, W. E., \& Heathcote, S. R. 1995, ApJ, 438, 724

Crotts, A. P. S. \& Yourdon, D. 2008, ApJ, 689, 1186

DeLaney, T., et al. 2010, ApJ, 725, 2038

Gallagher, J. S., et al. 2011, in Bulletin of the American Astronomical Society, Vol. 43, American Astronomical Society Meeting Abstracts \#217, 337.22-+

Garnavich, P. M., et al. 2001, in Bulletin of the American Astronomical Society, Vol. 33, American Astronomical Society Meeting Abstracts, 1370

Gouiffes, C., et al. 1988, A\&A, 198, L9

Hamuy, M., Suntzeff, N. B., Gonzalez, R., \& Martin, G. 1988, AJ, 95, 63

Havlen, R. J. 1972, A\&A, 16, 252

Kapteyn, J. C. 1902, Astronomische Nachrichten, 157, 201

Kjær, K., Leibundgut, B., Fransson, C., Jerkstrand, A., \& Spyromilio, J. 2010, A\&A, 517, A51

Krause, O., Birkmann, S. M., Usuda, T., Hattori, T., Goto, M., Rieke, G. H., \& Misselt, K. A. 2008a, Science, 320, 1195

Krause, O., Tanaka, M., Usuda, T., Hattori, T., Goto, M., Birkmann, S., \& Nomoto, K. 2008b, Nature, 456, 617

Liu, J.-F., Bregman, J. N., \& Seitzer, P. 2003, ApJ, 582, 919

Menzies, J. W., et al. 1987, MNRAS, 227, 39P

Miller, A. A., Smith, N., Li, W., Bloom, J. S., Chornock, R., Filippenko, A. V., \& Prochaska, J. X. 2010, AJ, 139, 2218

Nisenson, P. \& Papaliolios, C. 1999, ApJ, 518, L29

Ortiz, J. L., Sugerman, B. E. K., de La Cueva, I., Santos-Sanz, P., Duffard, R., Gil-Hutton, R., Melita, M., \& Morales, N. 2010, A\&A, 519, A7+

Otsuka, M., et al. 2012, ApJ, 744, 26

Patat, F. 2005, MNRAS, 357, 1161

Patat, F., Benetti, S., Cappellaro, E., \& Turatto, M. 2006, MNRAS, 369, 1949

Perrine, C. D. 1903, ApJ, 17, 310

Phillips, M. M., Hamuy, M., Heathcote, S. R., Suntzeff, N. B., \& Kirhakos, S. 1990, AJ, 99, 1133

Phillips, M. M., Heathcote, S. R., Hamuy, M., \& Navarrete, M. 1988, AJ, 95, 1087

Quinn, J. L., Garnavich, P. M., Li, W., Panagia, N., Riess, A., Schmidt, B. P., \& Della Valle, M. 2006, ApJ, 652, 512

Rest, A., et al. 2007, in Bulletin of the American Astronomical Society, Vol. 38, Bulletin of the American Astronomical Society, 935

Rest, A., et al. 2011a, ApJ, 732, 3

- 2008a, ApJ, 680, 1137

2012a, Nature, 482, 375

Rest, A., Sinnott, B., \& Welch, D. L. 2012b, PASA, 29, 466 
Rest, A., Sinnott, B., Welch, D. L., Foley, R. J., Narayan, G., Mandel, K., Huber, M. E., \& Blondin, S. 2011b, ApJ, 732, 2

Rest, A., et al. 2005a, ApJ, 634, 1103

- 2005b, Nature, 438, 1132

- 2008b, ApJ, 681, L81

Ritchey, G. W. 1901a, ApJ, 14, 293

-. 1901b, ApJ, 14, 167

- 1902, ApJ, 15, 129

Schaefer, B. E. 1987, ApJ, 323, L47

—. 1988, ApJ, 327, 347

Schmidt, B. P., Kirshner, R. P., Leibundgut, B., Wells, L. A., Porter, A. C., Ruiz-Lapuente, P., Challis, P., \& Filippenko, A. V. 1994, ApJ, 434, L19

Sinnott, B., Welch, D. L., Rest, A., Sutherland, P. G., \& Bergmann, M. 2013, ApJ, 767, 45

Smith, N., Davidson, K., Gull, T. R., Ishibashi, K., \& Hillier, D. J. 2003, ApJ, 586, 432

Sparks, W. B., Macchetto, F., Panagia, N., Boffi, F. R., Branch, D., Hazen, M. L., \& della Valle, M. 1999, ApJ, 523, 585

Sugerman, B. E. K. 2003, AJ, 126, 1939

- 2005, ApJ, 632, L17

Sugerman, B. E. K., et al. 2012, ApJ, 749, 170

Sugerman, B. E. K. \& Crotts, A. P. S. 2002, ApJ, 581, L97

Sugerman, B. E. K., Crotts, A. P. S., Kunkel, W. E., Heathcote, S. R., \& Lawrence, S. S. 2005a, ApJ, 627, 888

- 2005b, ApJS, 159, 60

Suntzeff, N. B., Hamuy, M., Martin, G., Gomez, A., \& Gonzalez, R. 1988a, AJ, 96, 1864

Suntzeff, N. B., Heathcote, S., Weller, W. G., Caldwell, N., \& Huchra, J. P. 1988b, Nature, 334, 135

Swope, H. H. 1940, Harvard College Observatory Bulletin, 913, 11

van den Bergh, S. 1965a, AJ, 70, 667

- 1965b, PASP, 77, 269

- 1966, PASP, 78, 74

- 1977, PASP, 89, 637

Van Dyk, S. D. 2013, ArXiv e-prints, 1305.6639

Van Dyk, S. D., Li, W., \& Filippenko, A. V. 2006, PASP, 118, 351

Wang, L., et al. 2002, ApJ, 579, 671

Wang, X., Li, W., Filippenko, A. V., Foley, R. J., Smith, N., \& Wang, L. 2008, ApJ, 677, 1060

Welch, D. L., Clayton, G. C., Campbell, A., Barlow, M. J., Sugerman, B. E. K., Meixner, M., \& Bank, S. H. R. 2007, ApJ, 669, 525

Westerlund, B. 1961, PASP, 73, 72

Wheeler, J. C., Maund, J. R., \& Couch, S. M. 2008, ApJ, 677, 1091

Whitelock, P. A., et al. 1988, MNRAS, 234, 5P

Xu, J., Crotts, A. P. S., \& Kunkel, W. E. 1994, ApJ, 435, 274

—. 1995, ApJ, 451, 806

Zwicky, F. 1940, Reviews of Modern Physics, 12, 66

\section{Discussion}

ZANARDO: Can the light echoes from SN 1987A shed some 'light' into the nature of the explosion and the likely asymmetric explosion?

REsT: Yes, with the data in hand, we already see significant asymmetry in H-alpha. We still have to put this more carefully into the context of the asymmetry in the ejecta and original CS dust structure.

Podsiadlowski: Is there a way your light-echo analysis could shed light on the issue of the mystery spot that has been reported in SN87A? 
REST: It depends on how bright the mystery spot is compared to the main explosion. If it is a significant fraction, then yes.

AndERSON: How many more events/transients do you think we will be able to apply this method to?

REST: Probably another 510 SN / eruptions in MW galaxy, and then also in some of the other Local Group galaxies like SMC and M31

MiLisAVlJEviC: Have you compared your results with the recent measurements of the 3D properties of SN 1987A's ejecta?

REST: We have just started to look more closely into this.

SANKRIT: With several years of experience now, is there some way of identifying Dustsheets that would be likely places for observing light echoes

REST: Spitzer 8 micron images seem to correlate best with the light echoes. However, having an 8 micron structure doesn't mean that there are light echoes, since also the distance to the dust sheet needs to be just right. 